\title{
Uma análise da vantagem de jogar em casa nas duas principais divisões do futebol profissional brasileiro
}

CDD. 20.ed. 796.33

\author{
Lucas Gomes de ALMEIDA* \\ Márcio Lopes de OLIVEIRA* \\ Cristiano Diniz da SILVA*
}

*Faculdade Governador

\begin{abstract}
Resumo
A vantagem em jogar em casa (VC) vem sendo um importante objeto de estudo no futebol mundial. Vários pesquisadores procuram quantificar essa vantagem no futebol internacional, porém quando se trata de futebol brasileiro, poucos estudos se dedicaram à pesquisa nessa área. Devido a isso, o presente estudo teve como objetivo comparar a VC no Campeonato Brasileiro das Séries A e B. Foram analisadas todas as partidas nas condições de vitórias, empates e derrotas em casa, das temporadas 2003 a 2009, tanto na série A quanto na série B. Para quantificar a VC, foi utilizada a metodologia de aproveitamento percentual de pontos, proposto por Pollard (1986). Foi encontrada uma VC maior na Série $B(69 \pm 2,3 \%)$ em relação à Série $A(65 \pm 2,3)(p<0,05)$. Pôde-se concluir que, na Série B do Campeonato Brasileiro, o fator "jogar em casa" exerce maior vantagem em relação à Série A Brasileira nas temporadas analisadas.
\end{abstract}

UnItERMos: Vantagem em casa; Campeonato Brasileiro; Futebol.

\section{Introdução}

Quando se discutem esportes coletivos e individuais, a vantagem em casa (VC) tem se tornado um fator bem explorado (CARRON, LOUGHHEAD \& Bray, 2005; Nevil \& Holder, 1999; Pollard, 2006), sendo bastante retratado desde as primeiras disputas do futebol inglês do final do século XIX (Pollard \& Pollard, 2005a). Uma metodologia simples para essa quantificação em uma determinada liga, introduzida inicialmente por POLLARD (1986), é através do cálculo da porcentagem de pontos obtidos pelas equipes mandantes, incluindo empates. Em uma competição com disputa equilibrada em que cada equipe joga duas vezes, uma em casa e outra fora, um desempenho porcentual maior que $50 \%$ é definido como evidência de que há VC.

NeVill e Holder (1999), em seu recente estudo, indentificaram quatro principais causas que podem ser responsáveis pela VC. Eles citaram a torcida, o privilégio arbitral e a familiaridade com o campo de jogo por parte do mandante, bem como as viagens efetuadas pelos visitantes, como fatores que podem ser aplicados na tentativa de explicação desse fenômeno na maioria dos esportes.

$\mathrm{Na}$ Inglaterra, o comportamento da $\mathrm{VC}$ no futebol é bem definido em diferentes épocas e divisões da liga (Clarke \& Norman, 1995; Nevill, Newell \& Gale, 1996; Pollard \& Pollard, 2005a), e até mesmo em comparação entre continentes (Pollard, 2006). Atualmente encontram-se valores variando de 60-65\%, com diferenças não significativas entre a primeira e segunda divisão nos países da Europa (Dosseville, 2007; Pollard \& Pollard, 2005a, 2005b). No Brasil, os estudos de Silva e Moreira (2008) e Pollard, Silva e Medeiros (2008) apontam que os valores encontrados ( $-65 \%)$ são maiores que os das principais ligas da Europa. Todavia, dados da VC do futebol brasileiro, comparando a primeira e segunda divisão, são limitados na literatura. Assim, o presente estudo objetivou comparar a VC no Campeonato Brasileiro da Série A e B. 


\section{Procedimentos metodológicos}

\section{Amostra}

Foram analisadas 5.497 partidas nas condiçōes de vitórias; empates e derrotas em casa dos Campeonatos Brasileiros da Série A e B de 2003 a 2009 (TABELA 1). Todas adotaram o critério de três pontos por vitória, um ponto para empate e zero ponto para derrota. As tabelas das ligas foram consultadas via internet, no site www. soccerway.com. Este site tem sido utilizado em estudos prévios para cálculo da VC (Pollard, 2006; Pollard, Silva \& Medeiros, 2008; SiLVA \& Moreira, 2008).

\section{Métodos}

Para quantificação da VC, foi utilizada a metodologia do aproveitamento percentual de pontos em casa, proposta por POllard (1986). Considera-se que há VC quando o aproveitamento é maior que 50\%.

\section{Análise estatística}

Os dados são apresentados como média \pm desviopadrão. Pressuposiçōes de normalidade foram verificadas por Shapiro-Wilks "test". Para comparação entre a primeira e segunda divisão, empregou-se o Student's "paired t-test" para controlar qualquer mudança anual. "Effect sizes" $\left(\eta^{2}\right)$ foram calculados para estimar a magnitude das diferenças e valores de 0,01, 0,06 e maiores que 0,14 foram considerados como pequeno, médio e grande, respectivamente (COHEN, 1988). Todas as análises estatísticas foram realizadas pelo "software" Statistical Package for the Social Sciences (SPSS $® 17$ for Windows, Chicago, IL, EUA). Considerou-se o valor de $\mathrm{p}<0,05$ para nível de significância.

TABELA 1 - J ogos analisados na Série A ena Série B do Campeonato Brasileiro de futebol entreas temporadas de 2003 a 2009.

\begin{tabular}{ccccc}
\hline \multirow{2}{*}{ Ano } & \multicolumn{2}{c}{ Série A } & \multicolumn{2}{c}{ Série B } \\
\cline { 2 - 5 } & Jogos & Equipes & Jogos & Equipes \\
\hline 2003 & 552 & 24 & 312 & 24 \\
2004 & 552 & 24 & 312 & 24 \\
2005 & 462 & 22 & 267 & 22 \\
2006 & 380 & 20 & 380 & 20 \\
2007 & 380 & 20 & 380 & 20 \\
2008 & 380 & 20 & 380 & 20 \\
2009 & 380 & 20 & 380 & 20 \\
Total & 3086 & & 2411 & \\
\hline
\end{tabular}

Valores da VC são expressos em média \pm desvio-padrão. * Significativamente diferente $(P<0,05)$.

\section{Resultados}

Os resultados obtidos indicaram uma VC significantemente maior $(\mathrm{p}<0,05)$ no Campeonato Brasileiro da Série B $(69 \pm 2,3 \%)$ em relação à Série

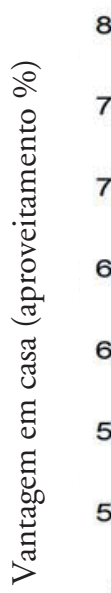

A $(65 \pm 2,3)$ (FIGURA 1). "Effect Size" foi calculado e revelou uma grande força $\left(\eta^{2}=0,57\right)$ de relevância dessa comparação.

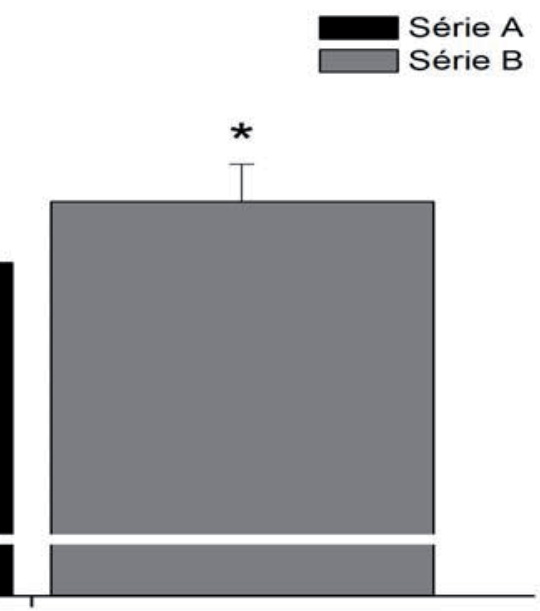

FIGURA 1 - Vantagem em casa (VC) registrada para o Campeonato Brasileiro da Série A e da Série B, comparada entre as temporadas de 2003 a 2008. 


\section{Discussão}

O principal objetivo do estudo foi comparar a VC no Campeonato Brasileiro da Série A e B durante as temporadas de 2003 a 2009. Os resultados revelaram que a VC na Série B foi maior que na Série A (FIGURA 1). Outros estudos que objetivaram comparar também a primeira e segunda divisão não denotaram diferenças significativas como, por exemplo, na liga inglesa (Pollard \& Pollard, 2005a, 2005b), na francesa (Dosseville, 2007; Pollard \& Pollard, 2005b), e nas ligas espanhola (Pollard \& Pollard, 2005b; Sanchez, García-Calvo, Leo, Pollard \& GÓmez, 2009), italiana e alemã (Pollard \& Pollard, 2005b). Conforme apontam Silva e Moreira (2008), o Brasil possui características diferenciadas em relação às principais ligas da Europa (ex. tamanho territorial, clima, calendário esportivo e perfil de torcedores) que podem causar diferentes impactos na VC. Isso fica demonstrado com as altas taxas de VC (>65\%) observados para as duas divisões em relação às principais ligas da Europa, que estão em torno de 61\%.

Desde o estudo clássico de SCHWARTZ e BARSKY (1977), diversas teorias têm surgido como tentativa de explicar quais mecanismos potenciais podem conferir às equipes mandantes tal vantagem. No caso do futebol, diversos estudos usando dados de várias competições domésticas, principalmente na Inglaterra, têm analisado esses fatores. Os resultados de alguns estudos são afirmativos e outros negativos para alguns dos fatores levantados e poderiam ter pesos diferenciados para o Brasil e neste, ainda, diferenciados para a Série A e B. Por exemplo, ao avaliar a relação entre $\mathrm{VC}$ e distância de deslocamento do visitante em competiçōes locais no futebol profissional, verifica-se que os estudos não encontraram resultados significativos (DowIE, 1982; POllard, 1986), ou mesmo tenderam a isso, mostrando uma relação pequena (Clark \& Norman, 1995) ( $\mathrm{r}=$ $0,07, p=0,0001$ ), considerando o grande número de observaçōes $(n=10,153$ jogos) que foram feitas.

Ao considerar as equipes que compuseram os campeonatos da Serie A e B nas temporadas analisadas no presente estudo, verifica-se que as equipes na Série A pode-se perceber que a grande maioria destas está situada nas regiōes sudeste (50\%) e sul (30\%) do país, com os outros $20 \%$ sendo divididos para as regiōes centro-oeste, nordeste e norte do Brasil. Enquanto isso, na Série B percebe-se que é um pouco mais "nacionalizado", pois não há uma grande concentração de equipes de uma mesma região. Assim, as evidências apontadas por Pollard, SiLVA e Medeiros (2008) de que a VC aumentou conforme aumenta a distância de deslocamento $(0,115 \mathrm{de}$ gol a favor do time da casa para cada $1000 \mathrm{~km}$ viajados pelo visitante) no campeonato Brasileiro da Série A da temporada de 2003 a 2007. Dessa forma, a maior taxa de VC na Série B poderia ser explicada parcialmente pelo fato de haver maiores deslocamentos e piores condições de viagens em relação aos times da Série A.

Tendo como objetivo de investigação os aspectos como tamanho e densidade de torcidas nos locais dos jogos de futebol, certos estudos têm concentrado suas hipóteses explicativas de VC na melhoria do desempenho das equipes mandantes em favor das decisōes favoráveis de árbitros (BОYKO, BОYKO \& BoyKo, 2007; Nevil, Balmer \& Williams, 2002; NeVIL \& NeWELl, 1996), por percepção de influência dos próprios torcedores (WOLFSON, WAKELIN \& LEWIS, 2005) e por sua influência positiva nos jogadores locais (Waters \& LovelL, 2002). Toda atmosfera criada pela torcida, salvo algumas exceções, pode criar um positivismo psicológico nos jogadores da casa, sendo considerado que, se os jogadores acreditarem na existência de VC, então é provável que aumente a confiança deles ao jogar em casa, e isso, consequentemente, contribui para a existência desse fenômeno (Nevil \& Holder, 1999; Pollard \& Pollard, 2005b; Waters \& Lovell, 2002).

No entanto, talvez por falta de semelhança de critérios metodológicos, alguns estudos que consideram somente a relação entre quantidade de torcida e VC têm demonstrado que esse fator isolado pode não ser predisponente de uma maior $\mathrm{VC}$ no futebol profissional. Alguns exemplos disso são os estudos em que não se encontraram diferenças de valores de $\mathrm{VC}$ entre a primeira e a segunda divisão no futebol inglês (Clarke \& Norman, 1995; Dovie, 1982; Pollard, 1986; Pollard \& Pollard, 2005b) e no francês (DosSEVILLE, 2007), mesmo a primeira divisão tendo maior número de torcedores nos estádios nos mesmos períodos. Uma exceção é o estudo de NEVILL e NEWELl (1996), que encontrou relação positiva entre a média de quantidade de torcida e $\mathrm{VC}$ de cada divisão, mas sua amostragem foi extremamente pequena, avaliando somente a temporada de 1992-93 das principais divisōes inglesas e escocesas. Um estudo (Pollard, 1986) mais amplo da própria liga inglesa, entre 1888 e 1984, não evidenciou que densidade da torcida possa exercer influência sobre a VC nessa liga.

No campeonato da Série B existe o fato de os jogos serem disputados em estádios menores, passando 
assim a imagem de uma pressão maior da torcida contra a equipe adversária. Essa característica da Série B também pode ter criado uma atmosfera que potencializou uma maior resposta de $\mathrm{VC}$ nessa divisão. Um fator sobre o qual não há muita controvérsia na literatura são as questōes de familiaridade com o campo de jogo para explicar a VC no futebol, considerada pelos técnicos e atletas como o aspecto mais importante na VC (Wolfson, WAKeLIN \& LEWIS, 2005). Nota-se que na Série B as condições do campo de jogo são piores em comparação às da Série $\mathrm{A}$, e geralmente as equipes treinam nesse campo de jogo, o que poderia ampliar as vantagens de jogar em um campo conhecido, reforçando também a maior taxa de VC encontrada nessa divisão.

O conhecimento de determinadas condições específicas, como influência do vento, posições do sol e referências visuais adquiridas, pode trazer benefícios ao se preparar e disputar um jogo dentro do ambiente familiar (Dosseville, 2007; DowIE, 1982; Pollard, 1986; Pollard \& Pollard, 2005b), podendo refletir até $24 \%$ da VC (Pollard, 1986). Aspecto como as diferentes marcas esportivas das bolas utilizadas (providenciada pelo mandante) também condicionou vantagens em jogar em casa, sendo constatado que $7 \%$ da $\mathrm{VC}$ advêm dessa condição na liga francesa da primeira e da segunda divisão, em estudo das temporadas de 2002/03 a 2004/05 (Dosseville, 2007).

Como observado, o estudo limitou-se a avaliar o campeonato brasileiro apenas a partir da temporada de 2003, ano que passou a ser disputado no sistema

\section{Conclusão}

Ao se analisar os resultados é possível concluir que na série $B$ do campeonato brasileiro existe maior $\mathrm{VC}$ em relação à série $\mathrm{A}$. Fatores como diferenciação no tamanho do estádios e condições no campo de jogo e, principalmente, os transtornos advindos das distâncias percorridas parecem ser bons índicos que explicam essa maior taxa na Série B. de "rodízio duplo" na Série A. Na série B, essa forma foi incorporada de modo integral na disputa, somente na temporada de 2006. Em competiçóes anteriores, a adoção de sistema misto era comum, em fases classificatórias e eliminatórias, e havia até competiçôes com equipes só se enfrentando uma só vez (SANTIAGo Junior, 2006). A utilização de temporadas passadas também dificultaria comparações com outras ligas, além de ser notado que, em competições de caráter eliminatório, suas fases finais tendem a ter uma VC diferenciada (Nevil \& Holder, 1999; Pollard, 1986; Pollard $\&$ Pollard, 2005b).

Outro fator que tem se tornado comum no Campeonato Brasileiro dos últimos anos é o fato de que algumas equipes, por questóes financeiras e/ ou punitivas com perda de mando de jogo, levaram seus jogos para outro local que infringe, em teoria, perda de vantagem por não jogar na verdadeira casa. Mesmo sabendo desse aspecto limitante, o presente estudo avaliou todas as partidas levando em consideração a divulgação nominal da CBF para "time da casa” (clube que aparece primeiro no confronto). A intenção da utilização dessas temporadas em ambas as competiçôes (Séries A e B) foi aumentar o intervalo amostral reportando um número maior de temporadas e de partidas, até porque a $\mathrm{VC}$ observada para o futebol brasileiro da primeira divisão desde os anos de 1998 até temporadas mais recentes apresentou comportamento semelhante (Pollard, 2006; Pollard, Silva \& Medeiros, 2008; Silva \& Moreira, 2008). Outros estudos poderão controlar esses fatores.
Mesmo o futebol tendo uma importância extremamente grande perante a cultura brasileira, poucos são os estudos que buscam saber a verdadeira importância de determinados aspectos relacionados a VC, e consequentemente os benefícios trazidos à equipe $\mathrm{da}$ casa. Esses aspectos deverão ser analisados em futuros estudos sobre a perspectiva psicológica, geográfica e da familiaridade dos jogadores com o campo de jogo. 


\begin{abstract}
Home advantage in the two main divisions of the Brazilian professional soccer

The Home Advantage (HA) has been an important object of study in world football. Several researchers have sought to quantify HA in the international soccer, but when it comes to Brazilian soccer, few studies have been devoted to research in this area. The present study aimed at comparing the HA in the Brazilian Championship Series A and B. All the games were analyzed in terms of wins, draws and defeats at home, in the seasons from 2003 to 2009 in both series A and series B. To quantify the HA, we used the method of recovery percentage points, proposed by PollaRD (1986). There was a greater HA in Series B $(69 \pm 2.3 \%)$ compared to Series A $(65 \pm 2.3)(p<0.05)$. It was concluded that, in Series B of the Brazilian Championship, the factor "play at home" has greater advantage than in the Brazilian Series A season.
\end{abstract}

UnITERMS: Home advantage; Brazilian Championship; Football.

\title{
Referências
}

BOYKO, R.H.; BOYKO, A.R.; BOYKO, M.G. Referee bias contributes to home advantage in English premiership football. Journal of Sports Sciences, London, v.25, n.11, p.1185-94, 2007.

CARRON, A.V.; LOUGHHEAD, T.M.; BRAY, S.R. The home advantage in sport competitions: Courneya and Carron's (1992) conceptual framework a decade later. Journal of Sports Sciences, London, v.23, n.4, p.395-407, 2005.

CLARKE, S.; NORMAN, J.M. Home ground advantage of individual clubs in English soccer. The Statistician, London, v.44, n.4, p.509-21, 1995.

COHEN J. Statistical power analysis for the behavioral sciences. 2nd ed. Hillsdale: Lawrence Erlbaum, 1988.

DOSSEVILLE, F.E.M. Influence of ball type on home advantage in French professional soccer. Perceptual and Motor Skills, Missoula, v.104, n.2, p.347-51, 2007.

DOWIE J. Why Spain should win the World Cup? New Scientist, London, v.94, n.10, p.693-95, 1982.

NEVILL, A.M.; BALMER, N.J.; WILLIAMS, A.M. The influence of crowd noise and experience upon refereeing decisions in football. Psychology of Sport and Exercise, Amsterdam, v.3, n.4, p.261-72, 2002.

NEVILL, A.M.; HOLDER, R.L. Home advantage in sport: an overview of studies on the advantage of playing at home. Sports Medicine, Auckland, v.28, n.4, p.221-36, 1999.

NEVILL, A.M.; NEWELL, S.M.; GALE, S. Factors associated with home advantage in English and Scottish soccer matches.Journal of Sports Sciences, London, v.14, n.2, p.181-6, 1996.

POLLARD, R. Home advantage in soccer: a retrospective analysis. Journal of Sports Sciences, London, v.4, n.3, p.237-48, 1986. Worldwide regional variations in home advantage in association football. Journal of Sports Sciences, London, v.24, n.3, p.231-40, 2006.

POLLARD, R.; POLLARD, G. Long-term trends in home advantage in professional team sports in North America and England (1876-2003). Journal of Sports Sciences, London, v.23, n.4, p.337-50, 2005 a.

. Home advantage in soccer: a review of its existence and causes. International Journal of Soccer Science, v.3, n.1, p.28-38, 2005b.

POLLARD, R.; SILVA, C.D.; MEDEIROS, N.C. Home advantage in football in Brazil: differences between teams and the effects of distance traveled. Revista Brasileira de Futebol: Brazilian Journal of Soccer Science, Viçosa, v.1, n. 1, p.3-10, 2008. SÁNCHEZ, P.A.; GARCÍA-CALVO, T.; LEO, F.M.; POLLARD, R.; GÓMEZ, M.A. An analysis of home advantage in the top two Spanish professional football leagues. Perceptual and Motor Skills, Missoula, v.108, n.3, p.789-97, 2009.

SANTIAGO JUNIOR, J.R.S. Os arquivos do Campeonato Brasileiro. São Paulo: Panda Books, 2006.

SCHWARTZ, B.; BARSKY, S.F. The home advantage. Social Forces: International Journal of Social Research, Chapel Hill, v.55, n.3, p.641-61, 1977.

SILVA, C.D.; MOREIRA, D.G. A vantagem em casa no futebol: comparação entre o Campeonato Brasileiro e as principais ligas nacionais do mundo. Revista Brasileira de Cineantropometria e Desempenho Humano, Florianópolis, v.10, n.2, p.184-8, 2008. 
ALMEIDA, L.G.; OLIVEIRA, M.L. \& SILVA, C.D.

WATERS, A.; LOVELL, G. An examination of the homefield advantage in a professional English soccer team from a psychological standpoint. Football Studies, Los Angeles, v.5, n.1, p.46-59, 2002.

WOLFSON, S.; WAKELIN, D.; LEWIS, M. Football supporters' perceptions of their role in the home advantage. Journal of Sports Sciences, London, v.23, n.4, p.365-74, 2005.

ENDEREÇO

Lucas Gomes de Almeida

R. Pernambuco, 73 - B. Chiquito Gazola 36500-000 - Ubá - MG - BRASIL

e-mail: lucas_efi@hotmail.com
Recebido para publicação: 18/12/2009

Revisado: 23/06/2010

Aceito: 20/09/2010 